Christian S. Simonetia

iD https://orcid.org/0000-0001-6140-1981

Tiago S. B. Nocera ${ }^{a}$

iD https://orcid.org/0000-0003-1928-1634

Elcio O. Vianna ${ }^{\mathrm{a}}$

(iD) https://orcid.org/0000-0003-1902-6326

a Universidade de São Paulo, Faculdade de Medicina de Ribeirão Preto,

Departamento de Clínica Médica.

Ribeirão Preto, SP, Brasil.

Contato:

Elcio O. Vianna

E-mail:

evianna@fmrp.usp.br

Os autores declaram não haver conflito de interesses.

Este estudo foi financiado pelo Processo Fapesp 2010/50212-6. Tiago Nocera recebeu bolsa científica da PIBIC-USP, e Elcio Vianna recebeu bolsa de produtividade da CNPq 311851/2018-5 durante o desenvolvimento deste estudo.

Os autores declaram que este estudo não foi apresentado em eventos científicos e que não foi baseado em dissertação ou tese.

\section{Exposição prolongada a animais de laboratório está associada ao aumento de casos de asma}

\author{
Prolonged exposure to laboratory animals is \\ associated with increasing asthma cases
}

\section{Resumo}

Objetivo: descrever o resultado do acompanhamento de trabalhadores sensibilizados a animais de laboratório que prolongaram sua exposição. Métodos: após um período de aproximadamente 7 anos, entramos em contato com todos os indivíduos com sensibilização alérgica ocupacional detectada em estudo anterior. Um questionário foi aplicado para situação ocupacional atual, relação entre alergia e a decisão de deixar o trabalho ou exposição e para asma, sibilância, rinite, sintomas cutâneos e dispneia noturna. Resultados: dos 74 indivíduos com sensibilização ocupacional, 45 responderam ao questionáriona segunda avaliação e 37 ainda estavam expostos. Ao comparar os dados da primeira avaliação com os da avaliação atual, observou-se um aumento na frequência de asma. Na primeira avaliação, entre todos os sensibilizados $(n=74), 27,0 \%$ responderam sim a ambas as questões "Você tem ou já teve asma?" e "A asma foi diagnosticada por um médico?". Na segunda avaliação, 7 anos depois, dos 37 sujeitos que ainda estavam expostos, $51,3 \%$ responderam sim a essas questões (OR: 2,80; IC95\%: $1,23-6,38 ; p=0,013)$. Não houve mudança na frequência de respostas positivas às outras perguntas. Conclusão: os dados demonstram aumento da frequência de asma entre trabalhadores com sensibilização ocupacional que prolongam a exposição a animais de laboratório.

Palavras-chave: asma; diagnóstico; alergias ocupacionais; trabalho; saúde do trabalhador.

\begin{abstract}
Objective: to describe the follow-up evaluation of sensitized workers who prolonged their occupational exposure to laboratory animals. Methods: after a follow-up period of approximately 7 years, we contacted all individuals with occupational allergic sensitization detected in a previous study. A questionnaire was employed to assess present occupational status, relationship between allergy and decision on quitting job or exposure, and to assess asthma, wheezing, rhinitis, skin symptoms, and nocturnal dyspnea. Results: of the 74 individuals with occupational sensitization, 45 volunteers completed the questionnaire at the second evaluation and 37 were still exposed. By comparing the data from the first evaluation with data from the current evaluation, we observed an increase in asthma frequency. In the first evaluation, among all sensitized subjects $(n=74), 27.0 \%$ answered yes to both questions "Do you have or have you ever had asthma?" and "Was the asthma diagnosed by a doctor?" In the second evaluation, 7 years later, among the 37 subjects who were still exposed, $51.3 \%$ answered yes to these questions (OR: 2.80; 95\%CI: 1.23-6.38; $p=0.013$ ). There was no change in the frequency of positive responses to the other questions. Conclusion: data demonstrate increasing frequency of asthma among workers with occupational sensitization who prolong exposure to laboratory animal.
\end{abstract}

Keywords: asthma; diagnosis; occupational allergies; work; occupational health. 


\section{Introdução}

Trabalhadores expostos a animais de laboratório frequentemente desenvolvem sintomas alérgicos, condição conhecida como alergia a animais de laboratório $(\mathrm{AAL})^{1}$, caracterizada por urticária, conjuntivite, rinite e asma ${ }^{2}$. A prevalência de AAL pode variar de 11 a 44\%, sendo que essa grande variação na prevalência se deve aos diferentes critérios de definição da AAL, com definições baseadas em relatos de sintomas ou exames laboratoriais ${ }^{3,4}$. Em todo o caso, AAL é um problema considerável na saúde ocupacional ${ }^{5}$.

AAL é causada por uma reação de hipersensibilidade imunológica a antígenos de alto peso molecular que estão presentes na urina, pelos e saliva de animais de laboratório ${ }^{6}$. Os principais antígenos eliminados por ratos capazes de gerar reações alérgicas são Rat n 1A e Rat $n$ 1B, que são antígenos variantes de alfa-2-globulinas; o principal antígeno do camundongo é uma pré-albumina chamada Mus $\mathrm{m}^{7}$. Ambos Rat n1 e Mus m1 são produzidos no fígado, sob o controle de hormônios androgênicos. Rat n1 é liberado em grandes quantidades por ratos machos adultos. Da mesma forma, camundongos machos adultos liberam até 4 vezes mais o alérgeno Mus $\mathrm{m} 1$ do que camundongos fêmeas ${ }^{8}$.

No ambiente de trabalho, o contato com antígenos eliminados por esses animais ocorre durante atividades rotineiras, como contato com fluidos corporais durante a limpeza das gaiolas, alimentação e transporte dos animais, coleta de tecidos, cirurgia, inoculação e sacrifícios. Além disso, esses antígenos podem ser encontrados suspensos no ar ou depositados em qualquer superfície ${ }^{9}$. Todas essas características tornam as salas de animais e laboratórios de pesquisa ambientes de trabalho que levam ao desenvolvimento de reações alérgicas a animais de laboratório ${ }^{10,11}$.

A sensibilização alérgica a animais de laboratório está associada a um risco crescente de sintomas cutâneos, dispneia noturna, rinite, sibilância, hiperresponsividade brônquica e asma, em comparação com a sensibilização alérgica a alérgenos comuns ${ }^{12}$. A sensibilização ocupacional, como importante fator de risco para AAL, chama muita atenção, uma vez que a prevalência da sensibilização a animais de laboratório varia de 16 a $25 \%{ }^{13,14}$.

Muitos dos indivíduos que se tornam sensibilizados aos animais de laboratório prolongam sua exposição aos animais devido à necessidade de manter o emprego ou de concluir suas atividades de pesquisa.

Em uma avaliação anterior (2010-2012), descrevemos a prevalência da sensibilização a animais de laboratório. Para este artigo, reavaliamos indivíduos que foram considerados sensibilizados. O objetivo deste estudo de acompanhamento foi avaliar e relatar o risco de sintomas alérgicos associados à exposição prolongada. Também descrevemos como a sensibilização anterior a animais alérgicos afetou a decisão dos indivíduos de abandonar a exposição a animais de laboratório.

\section{Métodos}

Este é um estudo prospectivo com avaliação de técnicos, estudantes e pesquisadores que atuam em laboratórios de animais. Para a primeira avaliação realizada de 2010 a 2012, foram inscritos 453 sujeitos. Cento e cinquenta e dois eram funcionários e 301 eram alunos de duas universidades brasileiras, Universidade de São Paulo (USP) em Ribeirão Preto e Universidade Estadual de Campinas (Unicamp) em Campinas.

Dentre esses 453 sujeitos, 74 voluntários (16,3\%) foram sensibilizados a pelo menos um animal de laboratório $^{12}$. A sensibilização foi definida por teste cutâneo positivo para um ou mais dos seguintes alérgenos: rato, camundongo, coelho, hamster ou cobaia.

Para esta análise todos os 74 indivíduos com sensibilização animal foram contatados por telefone, e-mail, rede social e por meio de busca no local de trabalho. Foi aplicado um questionário para avaliar a evolução, situação profissional atual, relação entre alergia e decisão de abandonar ou não aquele emprego ou exposição.

Para avaliação dos sintomas, foi utilizado o questionário European Community Respiratory Health Survey, um questionário autoaplicável traduzido para o português, adaptado para o léxico brasileiro e validado ${ }^{15}$. O estudo e o termo de consentimento foram revisados e aprovados pelo Comitê de Ética da Faculdade de Medicina de Ribeirão Preto da USP-RP (protocolo número 4674/2015). O Termo de Consentimento Livre e Esclarecido (TCLE) foi obtido de todos os indivíduos após a leitura e discussão do protocolo individualmente. Os voluntários possuíam boa formação cultural e eram capazes de compreender o TCLE sobre os riscos da exposição a animais de laboratório.

\section{Análise estatística}

A análise univariada (teste Qui-quadrado) foi realizada para comparar a frequência dos resultados entre a primeira e a segunda avaliação.

\section{Resultados}

Dos 74 profissionais com sensibilização ocupacional, 45 voluntários responderam ao questionário, 37 deles ainda estavam expostos a animais de laboratório até a data deste estudo. Perdemos dados de acompanhamento de 29 indivíduos porque $10 \mathrm{se}$ recusaram a responder nosso questionário, mesmo em seu formato reduzido que foi projetado para não aderentes, e 19 indivíduos não foram encontrados. O período médio de acompanhamento foi de 7 anos (5,0 - 7,5 anos). Na comparação dos dados das duas rodadas, observou-se aumento da frequência de asma, de $27,0 \%$ para $51,3 \%$ (OR: 2,80; IC95\%: 1,236,$38 ; \mathrm{p}=0,013$ ), com 7 novos casos de asma. Não houve mudança na frequência de sibilância, rinite, sintomas cutâneos e dispneia noturna (Tabela 1). 
Tabela 1 Comparação da frequência dos desfechos

\begin{tabular}{lcccc}
\hline Desfechos & $2^{a}$ avaliação vs $1^{a}$ avaliação & OR & IC95\% & p \\
\hline Asma & $51,3 \%$ vs $27,0 \%$ & 2,80 & $1,236,38$ & 0,013 \\
Sibilância & $40,5 \%$ vs 45,9\% & 0,74 & $0,331,68$ & 0,467 \\
Rinite & $86,4 \%$ vs 90,5\% & 0,72 & $0,192,72$ & 0,623 \\
Dispneia noturna & $37,8 \%$ vs 24,3\% & 1,86 & $0,794,36$ & 0,150 \\
Sintomas cutâneos & $48,6 \%$ vs 51,3\% & 0,95 & $0,42 \quad 2,13$ & 0,896 \\
\hline
\end{tabular}

OR: razão de possibilidades; IC: intervalo de confiança; $1^{\text {a }}$ avaliação $n=74 ; 2^{\text {a }}$ avaliação $n=37$.

Para verificar se os 45 sujeitos eram representativos de todo o grupo, foram feitas comparações em relação a características diferentes das variáveis de desfecho. Os resultados mostraram que sexo, idade, instituição (USP x Unicamp) e tipo de afiliação (pesquisadores, alunos ou técnicos) não foram diferentes na comparação do grupo de estudo atual com o grupo de estudo anterior. Uma comparação semelhante foi feita entre os 37 indivíduos que ainda estavam expostos e todo o grupo de 74 indivíduos, e nenhuma diferença foi detectada.

Entre os 37 indivíduos com exposição prolongada, 3 relataram vontade de desistir da exposição a animais de laboratório devido às informações clínicas fornecidas durante a primeira análise. Em relação aos resultados das avaliações de responsividade brônquica realizadas durante a primeira avaliação, 16 dos 37 indivíduos com exposição prolongada testaram positivo. Não detectamos um resultado claro ou consistente associado a um teste positivo. Dentre os 3 indivíduos dispostos a desistir da exposição, um testou positivo para broncoprovocação, ou seja, hiperresponsividade brônquica.

Entre os 8 indivíduos que abandonaram suas atividades relacionadas aos animais, dois apresentaram hiperresponsividade brônquica, mas relataram que os motivos para parar não foram associados a esses achados, sintomas ou outros resultados de testes. Todos os indivíduos tinham espirometria normal e, portanto, $\mathrm{VEF}_{1}$ normal.

Entre os 7 novos casos de asma, 6 indivíduos não apresentaram hiperresponsividade brônquica na primeira avaliação e desenvolveram asma ao longo do acompanhamento, e 2 casos com hiperresponsividade brônquica na primeira avaliação não foram considerados asmáticos naquele momento por não apresentarem sintomas.

\section{Discussão}

A prevalência de asma neste estudo é muito alta. Entre os brasileiros adultos, a prevalência de diagnóstico médico de asma é de $7,0 \%{ }^{16}$. Em estudo publicado em 2017, no qual analisamos a prevalência de desfechos em indivíduos com sensibilização comum e sensibilização ocupacional, mostramos que a prevalência de asma entre indivíduos com sensibilização comum foi de 9,8\%, e a prevalência de asma no grupo de sensibilização ocupacional foi de $27,0 \%{ }^{12}$. Já na avaliação atual dos indivíduos com sensibilização ocupacional, a prevalência de diagnóstico médico de asma foi de 51,3\%, 7 vezes mais do que a prevalência geral de asma em adultos.

Estudos anteriores ${ }^{17,18}$ já haviam apresentado os efeitos deletérios da exposição continuada ao alérgeno ocupacional ao qual o indivíduo está sensibilizado. Palmberg et al. ${ }^{17}$ observaram que no $12^{\circ}$ mês de exposição, indivíduos que se tornaram sensibilizados a animais de laboratório relataram mais sintomas nasais e oftalmológicos em comparação com indivíduos não sensibilizados. Além disso, esses autores ${ }^{17}$ observaram que no $24^{\circ}$ mês de exposição, indivíduos com sensibilização animal apresentavam menor $\mathrm{VEF}_{1}$ em comparação com indivíduos não sensibilizados. Portengen et al. ${ }^{18}$ investigaram a relação entre sensibilização e alterações na função pulmonar em trabalhadores sensibilizados e não sensibilizados a animais de laboratório. De acordo com a análise de regressão múltipla, a sensibilização foi associada a quedas excessivas no $\mathrm{VEF}_{1}$ de $83 \mathrm{ml} / \mathrm{ano}(\mathrm{p}<0,05)$, na CVF de $148 \mathrm{ml} / \mathrm{ano}(\mathrm{p}<0,01)$ e no FEF25-75\% de $7 \mathrm{ml} / \mathrm{s} /$ ano $(p=0,9)$ em trabalhadores que foram sensibilizados e continuamente expostos em comparação com trabalhadores continuamente expostos, mas não sensibilizados. Os autores não descreveram valores espirométricos ou reduções por grupo.

Com relação à reatividade brônquica, Renstron et al. ${ }^{19}$ mostraram que ao final do período de acompanhamento (mediana de 18 meses), houve diferença de reatividade entre trabalhadores com sensibilização ocupacional, com dose média de metacolina sendo de 0,30 para uma queda de $20 \%$ do $\mathrm{VEF}_{1}$, enquanto no grupo não sensibilizado, a dose média de metacolina necessária para uma queda de $20 \%$ do $\mathrm{VEF}_{1}$ foi de 1,97.

Os dados publicados em estudos anteriores corroboram nossos achados neste estudo, mostrando que a exposição prolongada ao agente sensibilizante representa risco à saúde do trabalhador, ou seja, 
desenvolvimento de sintomas, alteração da função pulmonar e aumento da reatividade brônquica. Este estudo agrega dados sobre o aumento do número de asmáticos, embora um número limitado de indivíduos tenha participado da segunda avaliação. Curiosamente, vemos apenas um aumento nos casos de asma, que é o desfecho mais grave resultante da exposição a animais de laboratório. Esse fato nos leva a acreditar que a asma seja o percurso final das reações alérgicas causadas pela exposição a animais de laboratório.

Uma questão que chama a atenção nesta análise é a aparente falta de importância dada pelos voluntários à sensibilização ocupacional. Na primeira fase (há 7 anos), cada resultado obtido por esses estudos era encaminhado aos voluntários individualmente, em relatório escrito, e enviado rapidamente. Além disso, um resumo dos resultados foi enviado sem a identificação dos indivíduos à diretoria de cada unidade. Este estudo serviu para aumentar a conscientização sobre os riscos de alergia em cada instituição. Os casos positivos para qualquer diagnóstico (rinite e/ou asma) e sensibilização foram encaminhados para atendimento ambulatorial no respectivo hospital universitário.

Dos 8 voluntários que desistiram de se expor a animais de laboratório, nenhum o fez pela sensibilização ocupacional, e entre os 37 voluntários que continuaram expostos, apenas 3 pensaram em abandonar a exposição pela sensibilização ocupacional. Essa característica talvez se deva ao desconhecimento desses indivíduos sobre alergia a animais de laboratório. Isso pôde ser evidenciado na primeira avaliação do estudo antes do diagnóstico clínico, quando apenas $25,1 \%$ dos trabalhadores expostos a animais de laboratório relataram receber orientação verbal ou ter lido algo sobre os riscos da exposição de animais indutores de reações alérgicas ${ }^{13}$. Todos os indivíduos receberam os resultados da primeira avaliação, incluindo as informações sobre teste cutâneo positivo para alérgenos animais.

Fornecer informações e treinamento para práticas seguras em relação à exposição a animais de laboratório é de extrema importância, conforme demonstrado por Larese et al. ${ }^{14}$ após um programa de prevenção em que alguns de seus elementos consistem em educação e treinamento, no estabelecimento de boas práticas de trabalho para redução da exposição a animais de laboratório, uso de equipamentos de proteção respiratória, teste cutâneo com alérgenos ocupacionais e avaliação médica. Após essas práticas, houve redução na prevalência de sensibilização a animais de laboratório, de 25,6\% entre 2001 e 2004 para $8,2 \%$ entre 2013 e $2016^{14}$.

Por fim, os dados deste estudo demonstram os riscos da exposição prolongada a animais de laboratório. Acreditamos que esse grupo de trabalhadores deva ser submetido periodicamente a teste cutâneo com alérgenos ocupacionais. Em caso de sensibilização ocupacional, os trabalhadores devem ser alertados sobre o risco de permanecerem expostos aos animais. Além disso, acreditamos que programas de prevenção seriam muito úteis, pois poderiam destacar os riscos da exposição a animais de laboratório e reduzir a frequência da sensibilização.

\section{Contribuições dos autores}

Os autores contribuíram igualmente para a coleta e análise de dados, redação e revisão do manuscrito e aprovação da versão final e assumem total responsabilidade pelo estudo e o conteúdo aqui publicado.

\section{Referências}

1. Wolfle TL, Bush RK. The science and pervasiveness of laboratory animal allergy. ILAR J. 2001;42(1):1-3.

2. Hunskaar S, Fosse RT. Allergy to laboratory mice and rats: a review of the pathophysiology, epidemiology and clinical aspects. Lab Anim. 1990;24(4):358-74.

3. Acton D, McCauley L. Laboratory animal allergy: an occupational hazard. AAOHN J. 2007;55(6):241-4.

4. Bush RK, Stave GM. Laboratory animal allergy: an update. ILAR J. 2003;44(1):28-51.

5. Jeal H, Jones M. Allergy to rodents: an update. Clin Exp Allergy. 2010;40(11):1593-601.
6. Corradi M, Ferdenzi E, Mutti A. The characteristics, treatment and prevention of laboratory animal allergy. Lab Anim. 2012;42(1):26-33.

7. Wood RA. Laboratory animal allergens. ILAR J. 2001;42(1):12-6.

8. Jones M. Laboratory animal allergy in the modern era. Curr Allergy Asthma Rep. 2015;15(12):73.

9. Harries MG, Cromwell O. Occupational asthma caused by allergy to pigs' urine. Br Med J. 1982;284(6319):867.

10. Bush RK, Wood RA, Eggleston PA. Laboratory animal allergy. J Allergy Clin Immunol. 1998;102(1):99-112. 
11. Taylor AN, Longbottom JL, Pepys J. Respiratory allergy to urine proteins of rats and mice. Lancet. 1977;2(8043):847-9.

12. Simoneti CS, Ferraz E, Menezes MB, Bagatin E, Arruda LK, Vianna EO. Allergic sensitization to laboratory animals is more associated with asthma, rhinitis, and skin symptoms than sensitization to common allergens. Clin Exp Allergy. 2017;47(11):1436-44.

13. Ferraz E, Arruda LKP, Bagatin E, Martinez EZ, Cetlin AA, Simoneti CS, et al. Laboratory animals and respiratory allergies: the prevalence of allergies among laboratory animal workers and the need for prophylaxis. Clinics. 2013;68(6)750-9.

14. Larese FF, Drusian A, Mauro M, Negro C. Laboratory animal allergy reduction from 2001 to 2016: an intervention study. Respir Med. 2018;136:71-6.

15. Ribeiro M, Angelini L, Robles-Ribeiro PG, Stelmach R, Santos UP, Terra-Filho M. Validation of the Brazilian-Portuguese version of the European
Community Respiratory Health Survey in asthma patients. J Asthma. 2007;44(5):371-5.

16. Vianna EO, García CA, Bettiol H, Barbieri MA, Rona RJ. Asthma definitions, relative validity and impact on known risk factors in young Brazilians. Allergy. 2007;62(10):1146-51.

17. Palmberg L, Sundblad B, Lindberg A, Kupczyk M, Sahlander K, Larsson K. Long term effect and allergic sensitization in newly employed workers in laboratory animal facilities. Respir Med. 2015;109(9):1164-73.

18. Portengen L, Hollander A, Doekes G, Meer G, Heederik D. Lung function decline in laboratory animal workers: the role of sensitization and exposure. Occup Environ Med. 2003;60(11):870-5.

19. Renstrom A, Malmberg P, Larsson K, Larsson PH, Sundblad BM. Allergic sensitization is associated with increased bronchial responsiveness: a prospective study of allergy to laboratory animals. Eur Respir J. 1995;8(9):1514-9. 